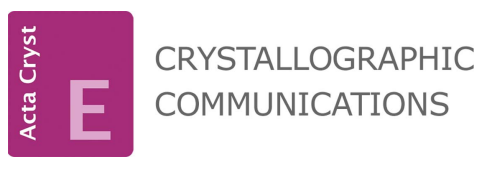

ISSN 2056-9890

Received 14 October 2019

Accepted 22 October 2019

Edited by J. Simpson, University of Otago, New Zealand

Keywords: crystal structure; benzoquinoline; nicotinamide derivative; photocyclization.

CCDC reference: 1960760

Supporting information: this article has supporting information at journals.iucr.org/e

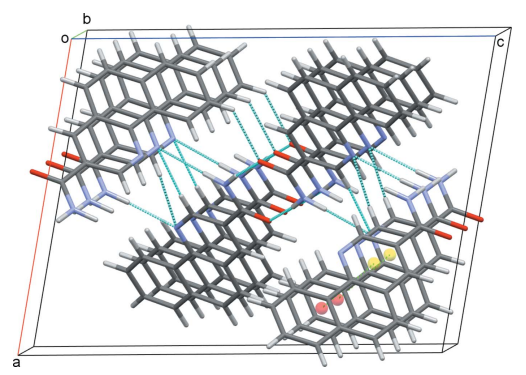

\section{Crystal structure of benzo[h]quinoline-3-carbox- amide}

\author{
Christoph W. Grathwol, ${ }^{a}$ Nicolas Chrysochos, ${ }^{\mathrm{b}}$ Benedict J. Elvers, ${ }^{\mathrm{b}}$ Andreas Link ${ }^{\mathrm{a} *}$ \\ and Carola Schulzke ${ }^{\mathrm{b} *}$
}

a Institut für Pharmazie, Universität Greifswald, Friedrich-Ludwig-Jahn-Strasse 17, 17489 Greifswald, Germany, and b Institut für Biochemie, Felix-Hausdorff-Strasse 4, 17489 Greifswald, Germany. *Correspondence e-mail: link@uni-greifswald.de, carola.schulzke@uni-greifswald.de

The title compound, $\mathrm{C}_{14} \mathrm{H}_{10} \mathrm{~N}_{2} \mathrm{O}$, crystallizes in the monoclinic space group $P 2_{1} / c$ with four molecules in the unit cell. All 17 non-H atoms of one molecule lie essentially in one plane. In the unit cell, two pairs of molecules are exactly coplanar, while the angle between these two orientations is close to perfectly perpendicular at $87.64(6)^{\circ}$. In the crystal, molecules adopt a 50:50 crisscross arrangement, which is held together by two nonclassical and two classical intermolecular hydrogen bonds. The hydrogen-bonding network together with off-centre $\pi-\pi$ stacking interactions between the pyridine and outermost benzene rings, stack the molecules along the $b$-axis direction.

\section{Chemical context}

Quinoline and benzoquinoline scaffolds are common structural motifs in artificial, as well as natural products, and many of these compounds are of enormous value for pharmacotherapy. Their multifaceted biological efficacy is outstanding and ranges from cardiovascular (Ferlin et al., 2002; Abouzid et al., 2008) and anti-inflammatory effects (Kumar et al., 2009; Hussaini, 2016) to antimicrobial (El Shehry et al., 2018), as well as anticancer activity (Abdelsalam et al., 2019; Haiba et al., 2019; Jafari et al., 2019; Musiol, 2017; Marzaro et al., 2016). In a report on 3-(tetrazol-5-yl)quinolines with antiallergic potential, benzo[ $h]$ quinoline-3-carboxamide was mentioned as a synthetic intermediate, though its biological activity was not determined in that work (Erickson et al., 1979). In our recent studies on photoswitchable sirtuin inhibitors, we obtained benzo $[h]$ quinoline-3-carboxamide as a side product of azastilbene photoisomerization (Grathwol et al., 2019). By UV radiation, $(E)$-5-styrylnicotinamide was transformed to its $Z$ isomer as envisioned, but underwent photocyclization and successive oxidation, yielding two isomeric benzoquinoline derivatives; the identity of one of these was determined to be the benzo[ $h]$ quinoline derivative and its crystal structure is reported here.<smiles>NC(=O)c1cnc2c(ccc3ccccc32)c1</smiles>$$
\text { Ported here. }
$$ 
Table 1

Hydrogen-bond geometry $\left(\AA,^{\circ}\right)$.

\begin{tabular}{lllll}
\hline$D-\mathrm{H} \cdots A$ & $D-\mathrm{H}$ & $\mathrm{H} \cdots A$ & $D \cdots A$ & $D-\mathrm{H} \cdots A$ \\
\hline $\mathrm{N} 1-\mathrm{H} 1 N \cdots \mathrm{N} 2^{\mathrm{i}}$ & $0.97(3)$ & $2.17(3)$ & $3.133(3)$ & $173(2)$ \\
$\mathrm{N} 1-\mathrm{H} 1 P \cdots \mathrm{O} 1^{\mathrm{ii}}$ & $0.93(3)$ & $1.96(3)$ & $2.895(3)$ & $175(3)$ \\
$\mathrm{C} 3-\mathrm{H} 3 \cdots \mathrm{N} 2^{\mathrm{i}}$ & 0.95 & 2.41 & $3.361(3)$ & 174 \\
$\mathrm{C} 7-\mathrm{H} 7 \cdots \mathrm{O} 1^{\mathrm{iii}}$ & 0.95 & 2.45 & $3.140(3)$ & 129 \\
\hline
\end{tabular}

Symmetry codes: (i) $-x+1, y-\frac{1}{2},-z+\frac{1}{2} ; \quad$ (ii) $\quad-x+1,-y-1,-z+1$; (iii) $x,-y+\frac{1}{2}, z-\frac{1}{2}$.

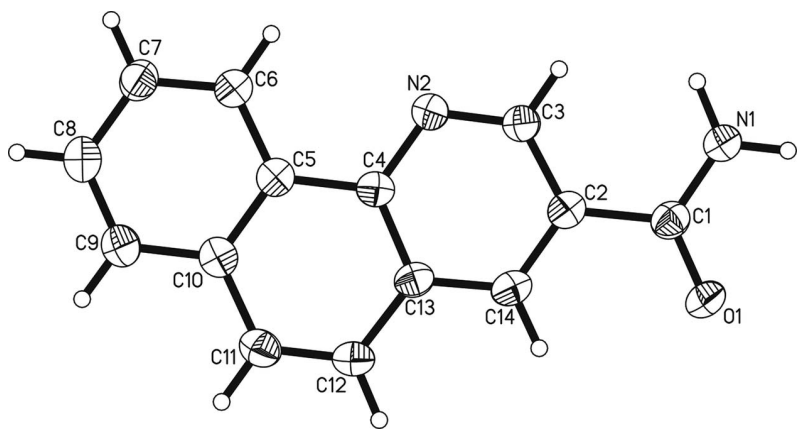

Figure 1

The molecular structure of benzo[ $h]$ quinoline-3-carboxamide. Displacement ellipsoids are shown at the $50 \%$ probability level.

\section{Structural commentary}

The title compound, benzo[ $h]$ quinoline-3-carboxamide, crystallizes in the monoclinic space group $P 2_{1} / c$. Four molecules are present in the unit cell $(Z=4)$ and there is one molecule in the asymmetric unit. Benzo[ $h]$ quinoline-3-carboxamide consists of a nicotinamide unit being fused with a benzo[ $h]$ quinoline moiety, while the pyridine ring is shared between these two common structural building blocks (Fig. 1). The molecule is essentially flat, with a largest deviation from the plane through all 17 non-H atoms of 0.050 (2) $\AA$ (O1) and an r.m.s. deviation of 0.020 (2) $\AA$. In the unit cell, the four molecules are arranged in two perfectly coplanar pairs, with a nearly perpendicular angle between the respective planes of the two pairs of $87.64(6)^{\circ}$ (Fig. 2). A plethora of crystal structures are known for compounds with one or other of the two building blocks that make up this molecule [for the nicotinamide scaffold, ConQuest finds over 2000 hits in the Cambridge Structural Database (CSD), while for benzoquinoline, there are over 500; Groom et al., 2016]. However,

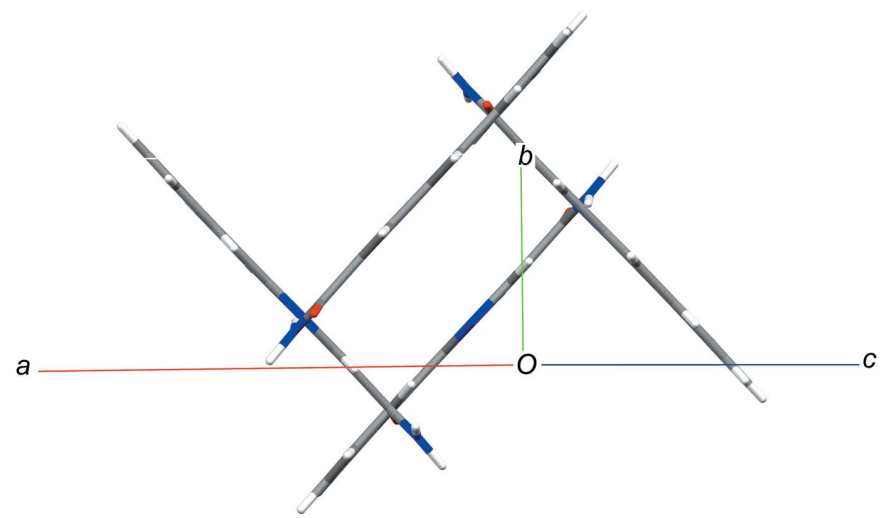

Figure 2

The unit cell of benzo[ $h$ ]quinoline-3-carboxamide in $P 2_{1} / c$, with its four molecules in a coplanar and perpendicular arrangement, viewed along the $a c$ diagonal.

the specific combination in the title compound is unprecedented. Comparing the title compound to the known structures of unsubstituted nicotinamides, its pronounced planarity is most notable. In the six published structures in the space groups $P 2_{1} / c$ or $P 2_{1} / a$, the angles between the aromatic plane (here $\mathrm{C} 2 / \mathrm{C} 3 / \mathrm{N} 2 / \mathrm{C} 4 / \mathrm{C} 13 / \mathrm{C} 14$ ) and the amide substituent (here O1/N1/C1) range from 22.1 to $23.3^{\circ}$ (general CSD refcode NICOAM; Wright \& King, 1954; Miwa et al., 1999; Fábián et al., 2011; Jarzembska et al., 2014), i.e. this angle is quite consistent. In the only distinct polymorph of a nicotinamide in the space group $P 2 / a$, four distinct molecules were refined with this angle ranging from 8.1 to $22.4^{\circ}$ (Li et al., 2011), i.e. they are not very consistent but still considerably larger than the corresponding angle found in the title compound, which is a mere $3.3(4)^{\circ}$. This points toward an extension of the aromatic resonance systems to include the amide substituent. In the parent nicotinamide scaffolds, this does not occur. Similarly, the comparatively long $\mathrm{C} 1=\mathrm{O} 1$ distance of $1.238(3) \AA$ (average $1.23 \AA$ ) and the comparatively short $\mathrm{C} 1-\mathrm{C} 2$ distance of 1.491 (3) $\AA$ (average $1.50 \AA$ in other nicotinamide structures) indicate some involvement of these atoms in resonance effects. In support of this extended resonance, in the nicotinamide structures, the aromatic $\mathrm{C}-\mathrm{C}$ bonds are much less diverse (range $1.38-1.39 \AA$, indicating very strong aromaticity in the pyridine ring) than in the structure reported here. In fact, the C-C [range 1.376 (3)-1.414 (3) $\AA$ ] and C$\mathrm{N}$ [1.321 (3) and 1.360 (3) $\mathrm{A}]$ bond lengths here are much

Figure 3

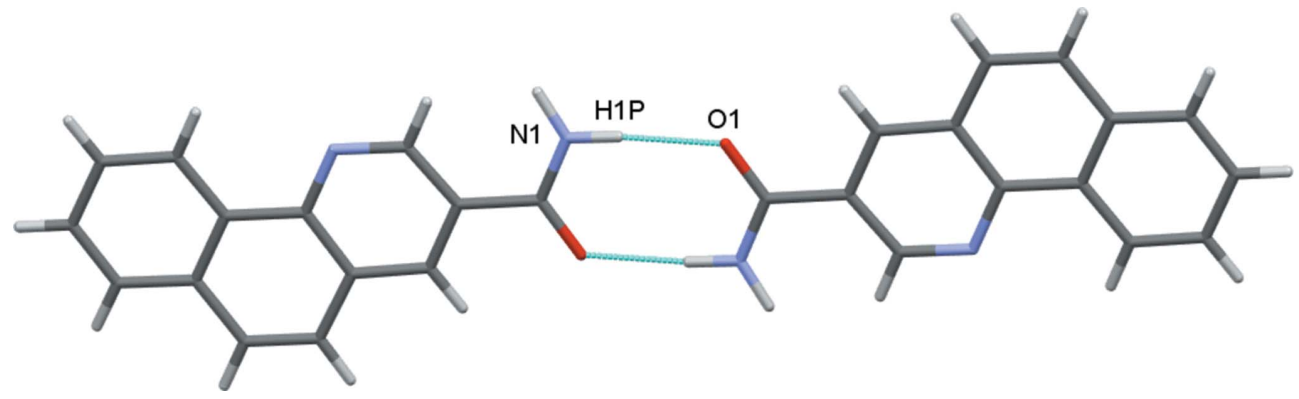

Dimers formed by $\mathrm{N}-\mathrm{H} \cdots \mathrm{O}$ hydrogen bonds. 


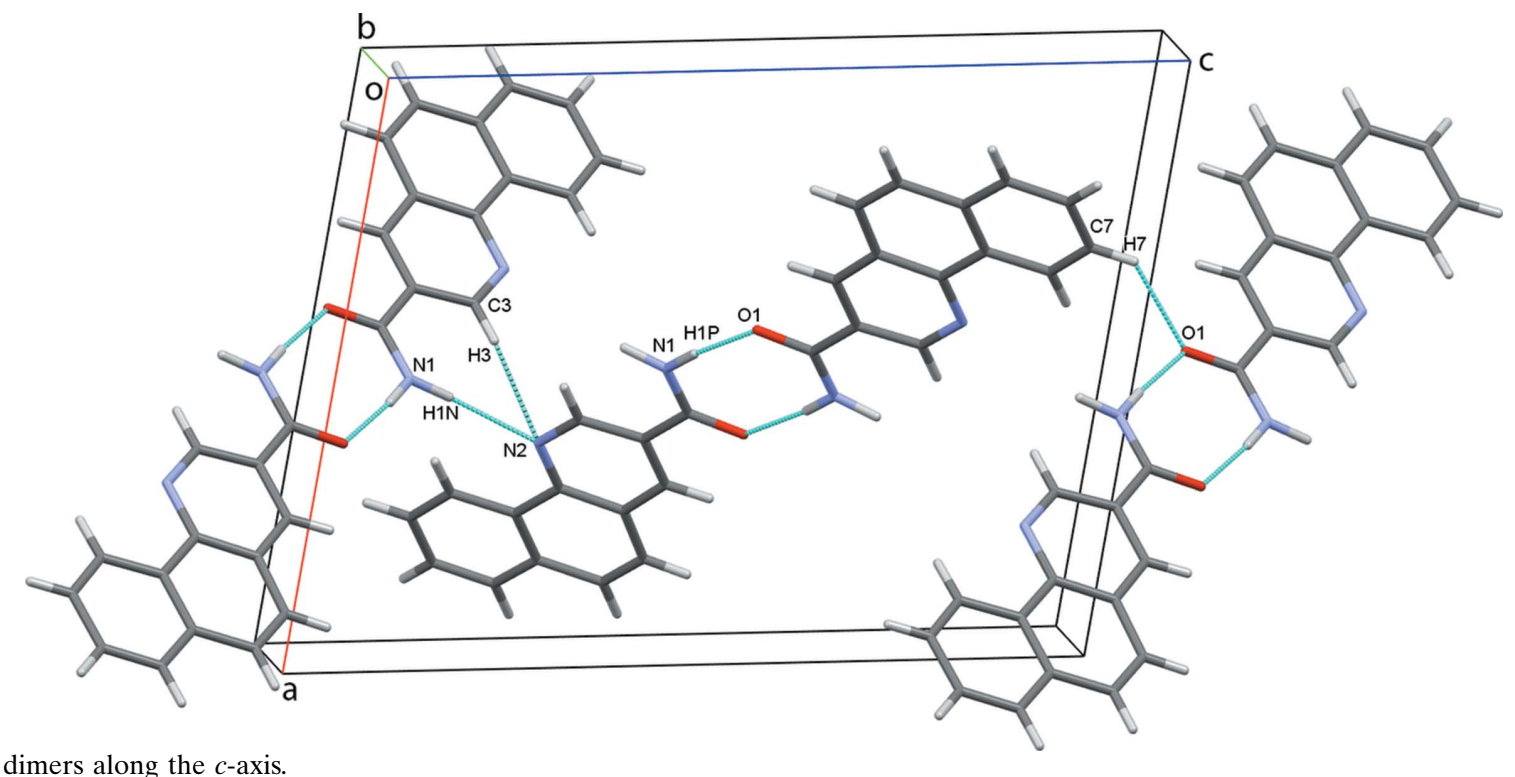

Figure 4

Chains of dimers along the $c$-axis.

more similar to the two known structures of 2-unsubstituted and 3-substituted benzo[ $h]$ quinolines (refcodes JAFVEU and SUDVES), with ranges of average $\mathrm{C}-\mathrm{C}$ and $\mathrm{C}-\mathrm{N}$ bond lengths of 1.38-1.42 and 1.32-1.36 ̊, respectively (Martínez et al., 1992; Luo et al., 2015). The benzo[h]quinoline structural motif therefore dominates the observed metrical parameters of the molecule reported here, representing a fusion between a nicotinamide and a benzo[ $h]$ quinoline, with a partial extension of the aromaticity beyond the ring system and extending towards the amide substituent.

\section{Supramolecular features}

In the crystal, the planar molecules are all arranged in planes in two distinct orientations, which are nearly perpendicular to each other [angle $87.64(6)^{\circ}$ ]. This forms a crisscross pattern

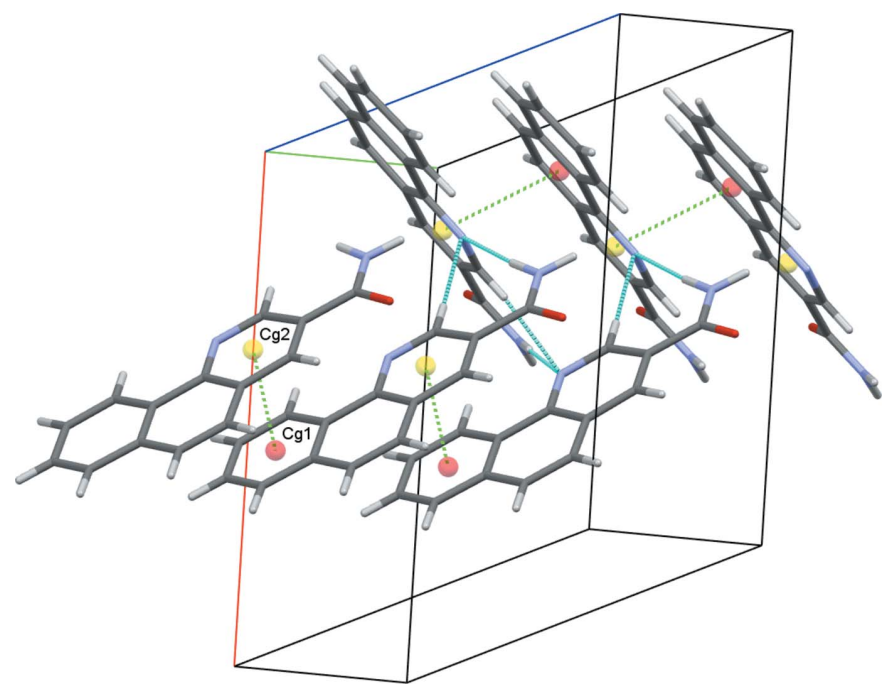

Figure 5

$\pi-\pi$ stacking interactions, with centroids shown as coloured spheres. $\mathrm{Cg} 1$ and $C g 2$ are the centroids of the $\mathrm{C} 5-\mathrm{C} 10$ and $\mathrm{C} 2 / \mathrm{C} 3 / \mathrm{N} 2 / \mathrm{C} 4 / \mathrm{C} 13 / \mathrm{C} 14$ rings, respectively. when viewed along the $a c$ diagonal (Fig. 2). Classical inversion-related $\mathrm{N} 1-\mathrm{H} 1 \mathrm{P}$. . O 1 hydrogen bonds form dimers and generate $R_{2}^{2}(8)$ ring motifs (Fig. 3). Each molecule forms two classical $(\mathrm{N}-\mathrm{H} \cdots \mathrm{O}$ and $\mathrm{N}-\mathrm{H} \cdots \mathrm{N})$ and two nonclassical $(\mathrm{C}-\mathrm{H} \cdots \mathrm{N}$ and $\mathrm{C}-\mathrm{H} \cdots \mathrm{O}$ ) hydrogen bonds (Table 1 ), and these contacts link adjacent dimers into zigzag chains along the $c$-axis direction (Fig. 4). The observed packing is further stabilized by off-centre $\pi-\pi$ stacking between the pyridine and outermost benzene rings of each of the coplanar layers [centroid-to-centroid distance $=3.610$ (1) $\AA$ ] (Fig. 5). These contacts combine to stack the molecules along the $b$-axis direction (Fig. 6).

\section{Synthesis and crystallization}

A solution of $(E)-5$-styrylnicotinamide $(673 \mathrm{mg}, 3.00 \mathrm{mmol}$, 1.00 equiv. $)$ in methanol $(350 \mathrm{ml})$ was treated with a solution

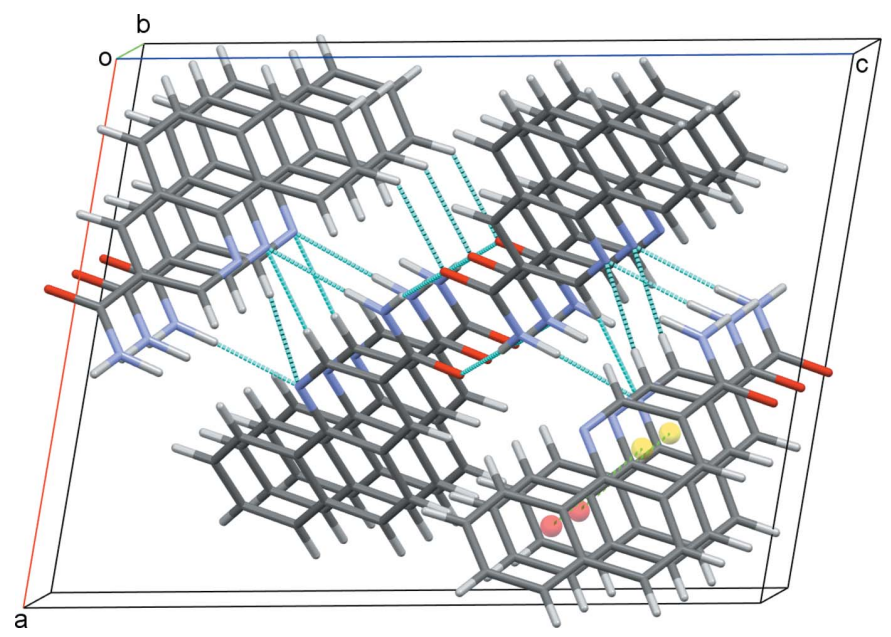

Figure 6

The overall packing of the title compound, viewed along the $b$-axis direction. 
of iodine (38 mg, $0.15 \mathrm{mmol}, 0.05$ equiv.) in methanol ( $50 \mathrm{ml}$ ). A slow stream of compressed air was bubbled through the reaction mixture while it was irradiated with UV light (six Vilber-Lourmat T8-C lamps, $8 \mathrm{~W}, 254 \mathrm{~nm}$ ). After complete consumption of the starting material $(24 \mathrm{~h})$, the solvent was removed under reduced pressure. Purification of the residue by silica-gel column chromatography ( $n$-hexane/THF, 1:1 $v / v$ ) gave pure benzo $[h]$ quinoline-3-carboxamide as a colourless solid (yield $80 \mathrm{mg}, 0.36 \mathrm{mmol}, 12 \%$ ). Crystallization was accomplished by slow evaporation of a solution in THF $\left(5 \mathrm{mg} \mathrm{ml}^{-1}\right)$ and yielded the title compound as colourless needles: $R_{\mathrm{F}}=0.32(n$-hexane/THF, $1: 1 v / v)$; m.p. $549.8 \mathrm{~K}$ (decomposition); ${ }^{1} \mathrm{H}$ NMR, H,H-COSY (400 MHz, DMSO$\left.d_{6}\right): \delta(\mathrm{ppm}) 9.48(d, J=2.2 \mathrm{~Hz}, 1 \mathrm{H}, \mathrm{C} 3-\mathrm{H}), 9.26-9.19(m, 1 \mathrm{H}$, C6-H), $8.89(d, J=2.1 \mathrm{~Hz}, 1 \mathrm{H}, \mathrm{C} 14-\mathrm{H}), 8.36(s, b r, 1 \mathrm{H}, \mathrm{N} 1-\mathrm{H})$, 8.12-8.07 ( $m, 1 \mathrm{H}, \mathrm{C} 9-\mathrm{H}), 8.03(d, J=8.9 \mathrm{~Hz}, 1 \mathrm{H}, \mathrm{C} 11-\mathrm{H}), 7.95$ $(d, J=8.9 \mathrm{~Hz}, 1 \mathrm{H}, \mathrm{C} 12-\mathrm{H}), 7.85-7.78(m, 2 \mathrm{H}, \mathrm{C} 7-\mathrm{H}, \mathrm{C} 8-\mathrm{H})$, $7.74(s, b r, 1 \mathrm{H}, \mathrm{N} 1-\mathrm{H}) ;{ }^{13} \mathrm{C}$ NMR, DEPT135, HSQC, HMBC (101 MHz, DMSO- $\left.d_{6}\right): \delta(\mathrm{ppm}) 166.4(\mathrm{C} 1), 147.8$ (C3), 146.7 (C4), 135.5 (C14), 133.8 (C13), 130.3 (C5), 129.0 (C8), 128.1 (C9/C11), 128.0 (C9/C11), 127.8 (C2), 127.3 (C7), 125.8 (C12), 124.9 (C10), 124.1 (C6); IR (ATR): $v\left(\mathrm{~cm}^{-1}\right)$ 3336, 3136, 1686, 1482, 1395, 1295, 801, 691, 539, 489; ESI-HRMS calculated for $\left[\mathrm{C}_{14} \mathrm{H}_{10} \mathrm{~N}_{2} \mathrm{O}+\mathrm{H}\right]^{+} 222.0793$, found 222.0796; compound purity (220 nm): $100 \%$.

\section{Refinement}

Crystal data, data collection and structure refinement details are summarized in Table 2. All C-bound $\mathrm{H}$ atoms constitute aromatic protons, which were attached in calculated positions and treated as riding with $U_{\text {iso }}(\mathrm{H})=1.2 U_{\text {eq }}(\mathrm{C})$. The two amine $\mathrm{H}$ atoms were found and refined without any constraints or restraints.

\section{Acknowledgements}

The authors acknowledge support for the Article Processing Charge from the DFG (German Research Foundation) and the Open Access Publication Fund of the University of Greifswald.

\section{Funding information}

Funding for this research was provided by: Deutsche Forschungsgemeinschaft (grant No. 393148499).

\section{References}

Abdelsalam, E. A., Zaghary, W. A., Amin, K. M., Abou Taleb, N. A., Mekawey, A. A. I., Eldehna, W. M., Abdel-Aziz, H. A. \& Hammad, S. F. (2019). Bioorg. Chem. 89, 102985.

Abouzid, K., Abdel Hakeem, M., Khalil, O. \& Maklad, Y. (2008). Bioorg. Med. Chem. 16, 382-389.

El Shehry, M. F., Ghorab, M. M., Abbas, S. Y., Fayed, E. A., Shedid, S. A. \& Ammar, Y. A. (2018). Eur. J. Med. Chem. 143, 1463-1473.

Erickson, H. E., Hainline, C. F., Lenon, L. S., Matson, C. J., Rice, T. K., Swingle, K. F. \& Van Winkle, M. (1979). J. Med. Chem. 22, 816-823.
Table 2

Experimental details.

\begin{tabular}{ll}
\hline Crystal data & \\
Chemical formula & $\mathrm{C}_{14} \mathrm{H}_{10} \mathrm{~N}_{2} \mathrm{O}$ \\
$M_{\mathrm{r}}$ & 222.24 \\
Crystal system, space group & Monoclinic, $P 2_{1} / c$ \\
Temperature $(\mathrm{K})$ & 170 \\
$a, b, c(\AA)$ & $12.634(3), 4.9426(10), 16.778(3)$ \\
$\beta\left({ }^{\circ}\right.$ & $100.53(3)$ \\
$V\left(\AA^{3}\right)$ & $1030.0(4)$ \\
$Z$ & 4 \\
Radiation type & Mo $K \alpha$ \\
$\mu\left(\mathrm{mm}^{-1}\right)$ & 0.09 \\
Crystal size $(\mathrm{mm})$ & $0.37 \times 0.07 \times 0.04$ \\
& \\
Data collection & \\
Diffractometer & Stoe IPDS-2T \\
Absorption correction & Numerical face indexed \\
$T_{\min }, T_{\max }$ & $0.727,0.997$ \\
No. of measured, independent and & $10053,2551,1320$ \\
$\quad$ observed $[I>2 \sigma(I)]$ reflections & \\
$R_{\text {int }}$ & 0.087 \\
(sin $\theta / \lambda)_{\text {max }}\left(\AA^{-1}\right)$ & 0.667 \\
Refinement & \\
$R\left[F^{2}>2 \sigma\left(F^{2}\right)\right], w R\left(F^{2}\right), S$ & \\
No. of reflections & $0.055,0.167,0.98$ \\
No. of parameters & 2551 \\
$\mathrm{H}$-atom treatment & 163 \\
& $\mathrm{H}$ atoms treated by a mixture of \\
$\Delta \rho_{\text {max }}, \Delta \rho_{\text {min }}\left(\mathrm{e} \AA^{-3}\right)$ & independent and constrained \\
\hline & refinement \\
& $0.23,-0.27$ \\
\hline
\end{tabular}

Computer programs: X-AREA (Stoe \& Cie, 2010), SHELXT2018 (Sheldrick, 2015a), SHELXL2018 (Sheldrick, 2015b), XP in SHELXTL (Sheldrick, 2008), Mercury (Macrae et al., 2008), CIFTAB (Sheldrick, 2015b) and publCIF (Westrip, 2010).

Fábián, L., Hamill, N., Eccles, K. S., Moynihan, H. A., Maguire, A. R., McCausland, L. \& Lawrence, S. E. (2011). Cryst. Growth Des. 11, 3522-3528.

Ferlin, M. G., Chiarelotto, G., Antonucci, F., Caparrotta, L. \& Froldi, G. (2002). Eur. J. Med. Chem. 37, 427-434.

Grathwol, C. W., Wössner, N., Swyter, S., Smith, A. C., Tapavicza, E., Hofstetter, R. K., Bodtke, A., Jung, M. \& Link, A. (2019). Beilstein J. Org. Chem. 15, 2170-2183.

Groom, C. R., Bruno, I. J., Lightfoot, M. P. \& Ward, S. C. (2016). Acta Cryst. B72, 171-179.

Haiba, M. E., Al-Abdullah, E. S., Ahmed, N. S., Ghabbour, H. A. \& Awad, H. M. (2019). J. Mol. Struct. 1195, 702-711.

Hussaini, S. M. A. (2016). Expert Opin. Ther. Pat. 26, 1201-1221.

Jafari, F., Baghayi, H., Lavaee, P., Hadizadeh, F., Soltani, F., Moallemzadeh, H., Mirzaei, S., Aboutorabzadeh, S. M. \& Ghodsi, R. (2019). Eur. J. Med. Chem. 164, 292-303.

Jarzembska, K. N., Hoser, A. A., Kamiński, R., Madsen, A., Durka, K. \& Woźniak, K. (2014). Cryst. Growth Des. 14, 3453-3465.

Kumar, S., Bawa, S. \& Gupta, H. (2009). Mini Rev. Med. Chem. 9, 1648-1654.

Li, J., Bourne, S. A. \& Caira, M. R. (2011). Chem. Commun. 47, 15301532.

Luo, C.-Z., Gandeepan, P., Wu, Y.-C., Chen, W.-C. \& Cheng, C.-H. (2015). RSC Adv. 5, 106012-106018.

Macrae, C. F., Bruno, I. J., Chisholm, J. A., Edgington, P. R., McCabe, P., Pidcock, E., Rodriguez-Monge, L., Taylor, R., van de Streek, J. \& Wood, P. A. (2008). J. Appl. Cryst. 41, 466-470.

Martínez, R., Toscano, R. A., Linzaga, I. E. \& Sánchez, H. (1992). J. Heterocycl. Chem. 29, 1385-1388.

Marzaro, G., Dalla Via, L., García-Argáez, A. N., Dalla Via, M. \& Chilin, A. (2016). Bioorg. Med. Chem. Lett. 26, 4875-4878.

Miwa, Y., Mizuno, T., Tsuchida, K., Taga, T. \& Iwata, Y. (1999). Acta Cryst. B55, 78-84. 
Musiol, R. (2017). Exp. Opin. Drug Discov. 12, 583-597.

Sheldrick, G. M. (2008). Acta Cryst. A64, 112-122.

Sheldrick, G. M. (2015a). Acta Cryst. A71, 3-8.

Sheldrick, G. M. (2015b). Acta Cryst. C71, 3-8.
Stoe \& Cie (2010). X-AREA. Stoe \& Cie GmbH, Darmstadt, Germany.

Westrip, S. P. (2010). J. Appl. Cryst. 43, 920-925.

Wright, W. B. \& King, G. S. D. (1954). Acta Cryst. 7, 283-288. 


\section{supporting information}

Acta Cryst. (2019). E75, 1828-1832［https://doi.org/10.1107/S2056989019014440]

\section{Crystal structure of benzo[h]quinoline-3-carboxamide}

\section{Christoph W. Grathwol, Nicolas Chrysochos, Benedict J. Elvers, Andreas Link and Carola \\ Schulzke}

Computing details

Data collection: $X$-AREA (Stoe \& Cie, 2010).; cell refinement: X-AREA (Stoe \& Cie, 2010).; data reduction: $X$-AREA (Stoe \& Cie, 2010).; program(s) used to solve structure: SHELXT2018 (Sheldrick, 2015a); program(s) used to refine structure: SHELXL2018 (Sheldrick, 2015b); molecular graphics: XP in SHELXTL (Sheldrick, 2008) and Mercury (Macrae et al., 2008); software used to prepare material for publication: CIFTAB (Sheldrick, 2015b) and publCIF (Westrip, 2010).

Benzo $[h]$ quinoline-3-carboxamide

Crystal data

$\mathrm{C}_{14} \mathrm{H}_{10} \mathrm{~N}_{2} \mathrm{O}$

$M_{r}=222.24$

Monoclinic, $P 2_{1} / c$

$a=12.634(3) \AA$

$b=4.9426(10) \AA$

$c=16.778(3) \AA$

$\beta=100.53(3)^{\circ}$

$V=1030.0(4) \AA^{3}$

$Z=4$

Data collection

Stoe IPDS2T diffractometer

Radiation source: fine-focus sealed tube Detector resolution: 6.67 pixels $\mathrm{mm}^{-1}$

$\omega$ scans

Absorption correction: numerical face indexed

$T_{\min }=0.727, T_{\max }=0.997$

Refinement

Refinement on $F^{2}$

Least-squares matrix: full

$R\left[F^{2}>2 \sigma\left(F^{2}\right)\right]=0.055$

$w R\left(F^{2}\right)=0.167$

$S=0.98$

2551 reflections

163 parameters

0 restraints

Primary atom site location: dual
$F(000)=464$

$D_{\mathrm{x}}=1.433 \mathrm{Mg} \mathrm{m}^{-3}$

Mo $K \alpha$ radiation, $\lambda=0.71073 \AA$

Cell parameters from 11960 reflections

$\theta=6.4-59.0^{\circ}$

$\mu=0.09 \mathrm{~mm}^{-1}$

$T=170 \mathrm{~K}$

Needle, colourless

$0.37 \times 0.07 \times 0.04 \mathrm{~mm}$

10053 measured reflections

2551 independent reflections

1320 reflections with $I>2 \sigma(I)$

$R_{\text {int }}=0.087$

$\theta_{\max }=28.3^{\circ}, \theta_{\min }=3.2^{\circ}$

$h=-16 \rightarrow 16$

$k=-6 \rightarrow 6$

$l=-22 \rightarrow 22$

Secondary atom site location: difference Fourier map

Hydrogen site location: mixed

$\mathrm{H}$ atoms treated by a mixture of independent and constrained refinement

$w=1 /\left[\sigma^{2}\left(F_{\mathrm{o}}^{2}\right)+(0.086 P)^{2}\right]$

where $P=\left(F_{\mathrm{o}}^{2}+2 F_{\mathrm{c}}^{2}\right) / 3$

$(\Delta / \sigma)_{\max }<0.001$

$\Delta \rho_{\max }=0.23$ e $\AA^{-3}$

$\Delta \rho_{\min }=-0.27 \mathrm{e} \AA^{-3}$ 
Extinction correction: SHELXL2018 (Sheldrick, 2015b),

$\mathrm{Fc}^{*}=\mathrm{kFc}\left[1+0.001 \mathrm{xFc}^{2} \lambda^{3} / \sin (2 \theta)\right]^{-1 / 4}$

Extinction coefficient: 0.019 (4)

\section{Special details}

Geometry. All esds (except the esd in the dihedral angle between two 1.s. planes) are estimated using the full covariance matrix. The cell esds are taken into account individually in the estimation of esds in distances, angles and torsion angles; correlations between esds in cell parameters are only used when they are defined by crystal symmetry. An approximate (isotropic) treatment of cell esds is used for estimating esds involving l.s. planes.

Fractional atomic coordinates and isotropic or equivalent isotropic displacement parameters $\left(\AA^{2}\right)$

\begin{tabular}{lllll}
\hline & $x$ & $y$ & $z$ & $U_{\text {iso }} / U_{\text {eq }}$ \\
\hline O1 & $0.60103(14)$ & $-0.2438(3)$ & $0.51392(9)$ & $0.0364(4)$ \\
N2 & $0.63400(15)$ & $0.2758(4)$ & $0.28016(11)$ & $0.0304(5)$ \\
N1 & $0.48278(17)$ & $-0.3250(4)$ & $0.39872(12)$ & $0.0331(5)$ \\
C1 & $0.56558(19)$ & $-0.1907(4)$ & $0.44191(13)$ & $0.0312(5)$ \\
C2 & $0.61839(18)$ & $0.0252(4)$ & $0.40098(12)$ & $0.0287(5)$ \\
C3 & $0.58725(19)$ & $0.0909(4)$ & $0.31880(13)$ & $0.0311(5)$ \\
H3 & 0.528010 & -0.004871 & 0.288573 & $0.037^{*}$ \\
C4 & $0.71867(17)$ & $0.4149(4)$ & $0.32278(12)$ & $0.0274(5)$ \\
C5 & $0.77008(18)$ & $0.6182(4)$ & $0.28123(13)$ & $0.0292(5)$ \\
C6 & $0.7362(2)$ & $0.6735(5)$ & $0.19826(13)$ & $0.0332(5)$ \\
H6 & 0.678537 & 0.573337 & 0.167687 & $0.040^{*}$ \\
C7 & $0.7858(2)$ & $0.8702(5)$ & $0.16151(14)$ & $0.0360(6)$ \\
H7 & 0.762331 & 0.905703 & 0.105414 & $0.043^{*}$ \\
C8 & $0.8707(2)$ & $1.0200(5)$ & $0.20521(15)$ & $0.0375(6)$ \\
H8 & 0.904089 & 1.157365 & 0.178843 & $0.045^{*}$ \\
C9 & $0.90571(19)$ & $0.9702(5)$ & $0.28530(14)$ & $0.0346(6)$ \\
H9 & 0.963477 & 1.073076 & 0.314661 & $0.041^{*}$ \\
C10 & $0.85689(18)$ & $0.7665(4)$ & $0.32529(13)$ & $0.0305(5)$ \\
C11 & $0.8935(2)$ & $0.7074(5)$ & $0.40961(14)$ & $0.0350(6)$ \\
H11 & 0.952662 & 0.805664 & 0.438992 & $0.042^{*}$ \\
C12 & $0.84594(19)$ & $0.5161(5)$ & $0.44794(13)$ & $0.0329(5)$ \\
H12 & 0.872211 & 0.481242 & 0.503736 & $0.039^{*}$ \\
C13 & $0.75657(17)$ & $0.3646(4)$ & $0.40605(12)$ & $0.0291(5)$ \\
C14 & $0.70365(19)$ & $0.1668(4)$ & $0.44428(13)$ & $0.0307(5)$ \\
H14 & 0.726853 & 0.130640 & 0.500358 & $0.037^{*}$ \\
H1N & $0.449(2)$ & $-0.279(6)$ & $0.344(2)$ & $0.059(9)^{*}$ \\
H1P & $0.452(2)$ & $-0.464(6)$ & $0.4246(16)$ & $0.054(8)^{*}$ \\
& & & & \\
\hline
\end{tabular}

Atomic displacement parameters $\left(\AA^{2}\right)$

\begin{tabular}{lllllll}
\hline & $U^{11}$ & $U^{22}$ & $U^{33}$ & $U^{12}$ & $U^{13}$ & $U^{23}$ \\
\hline O1 & $0.0445(10)$ & $0.0389(9)$ & $0.0247(8)$ & $-0.0018(8)$ & $0.0036(7)$ & $0.0056(7)$ \\
N2 & $0.0311(11)$ & $0.0302(10)$ & $0.0293(9)$ & $-0.0018(8)$ & $0.0037(8)$ & $0.0005(8)$ \\
N1 & $0.0373(12)$ & $0.0331(11)$ & $0.0280(10)$ & $-0.0018(9)$ & $0.0036(9)$ & $0.0020(8)$ \\
C1 & $0.0351(13)$ & $0.0302(11)$ & $0.0285(11)$ & $0.0031(10)$ & $0.0064(10)$ & $0.0001(9)$
\end{tabular}


supporting information

\begin{tabular}{lllllll} 
C2 & $0.0310(12)$ & $0.0281(11)$ & $0.0275(10)$ & $0.0048(9)$ & $0.0068(9)$ & $0.0004(9)$ \\
C3 & $0.0333(12)$ & $0.0306(11)$ & $0.0286(11)$ & $-0.0012(10)$ & $0.0033(9)$ & $0.0002(9)$ \\
C4 & $0.0278(11)$ & $0.0261(11)$ & $0.0279(11)$ & $0.0029(9)$ & $0.0036(9)$ & $-0.0021(9)$ \\
C5 & $0.0299(12)$ & $0.0276(11)$ & $0.0307(11)$ & $0.0034(10)$ & $0.0068(9)$ & $-0.0007(9)$ \\
C6 & $0.0345(13)$ & $0.0355(13)$ & $0.0295(11)$ & $-0.0024(10)$ & $0.0058(10)$ & $0.0009(9)$ \\
C7 & $0.0372(14)$ & $0.0387(13)$ & $0.0327(11)$ & $-0.0005(11)$ & $0.0082(10)$ & $0.0042(10)$ \\
C8 & $0.0367(13)$ & $0.0348(13)$ & $0.0428(13)$ & $-0.0015(11)$ & $0.0124(11)$ & $0.0025(11)$ \\
C9 & $0.0325(13)$ & $0.0324(12)$ & $0.0393(13)$ & $0.0003(10)$ & $0.0079(10)$ & $-0.0006(10)$ \\
C10 & $0.0291(12)$ & $0.0280(11)$ & $0.0351(11)$ & $0.0030(9)$ & $0.0079(9)$ & $-0.0021(9)$ \\
C11 & $0.0319(13)$ & $0.0379(13)$ & $0.0342(12)$ & $-0.0024(10)$ & $0.0037(10)$ & $-0.0063(10)$ \\
C12 & $0.0324(12)$ & $0.0372(12)$ & $0.0274(11)$ & $0.0009(10)$ & $0.0009(9)$ & $-0.0027(9)$ \\
C13 & $0.0303(12)$ & $0.0302(11)$ & $0.0260(10)$ & $0.0045(10)$ & $0.0033(9)$ & $0.0006(9)$ \\
C14 & $0.0352(13)$ & $0.0312(12)$ & $0.0248(10)$ & $0.0053(10)$ & $0.0034(9)$ & $0.0009(9)$ \\
\hline
\end{tabular}

Geometric parameters $\left(\AA,{ }^{\circ}\right)$

\begin{tabular}{|c|c|c|c|}
\hline $\mathrm{O} 1-\mathrm{C} 1$ & $1.238(3)$ & C6-H6 & 0.9500 \\
\hline $\mathrm{N} 2-\mathrm{C} 3$ & $1.321(3)$ & $\mathrm{C} 7-\mathrm{C} 8$ & $1.396(3)$ \\
\hline $\mathrm{N} 2-\mathrm{C} 4$ & $1.360(3)$ & $\mathrm{C} 7-\mathrm{H} 7$ & 0.9500 \\
\hline $\mathrm{N} 1-\mathrm{C} 1$ & $1.335(3)$ & $\mathrm{C} 8-\mathrm{C} 9$ & $1.358(3)$ \\
\hline $\mathrm{N} 1-\mathrm{H} 1 \mathrm{~N}$ & $0.97(3)$ & $\mathrm{C} 8-\mathrm{H} 8$ & 0.9500 \\
\hline $\mathrm{N} 1-\mathrm{H} 1 \mathrm{P}$ & $0.93(3)$ & $\mathrm{C} 9-\mathrm{C} 10$ & $1.413(3)$ \\
\hline $\mathrm{C} 1-\mathrm{C} 2$ & $1.491(3)$ & $\mathrm{C} 9-\mathrm{H} 9$ & 0.9500 \\
\hline $\mathrm{C} 2-\mathrm{C} 14$ & $1.376(3)$ & $\mathrm{C} 10-\mathrm{C} 11$ & $1.436(3)$ \\
\hline $\mathrm{C} 2-\mathrm{C} 3$ & $1.401(3)$ & $\mathrm{C} 11-\mathrm{C} 12$ & $1.346(3)$ \\
\hline $\mathrm{C} 3-\mathrm{H} 3$ & 0.9500 & $\mathrm{C} 11-\mathrm{H} 11$ & 0.9500 \\
\hline $\mathrm{C} 4-\mathrm{C} 13$ & $1.414(3)$ & $\mathrm{C} 12-\mathrm{C} 13$ & $1.428(3)$ \\
\hline $\mathrm{C} 4-\mathrm{C} 5$ & $1.443(3)$ & $\mathrm{C} 12-\mathrm{H} 12$ & 0.9500 \\
\hline $\mathrm{C} 5-\mathrm{C} 6$ & $1.406(3)$ & $\mathrm{C} 13-\mathrm{C} 14$ & $1.404(3)$ \\
\hline $\mathrm{C} 5-\mathrm{C} 10$ & $1.411(3)$ & $\mathrm{C} 14-\mathrm{H} 14$ & 0.9500 \\
\hline $\mathrm{C} 6-\mathrm{C} 7$ & $1.364(3)$ & & \\
\hline $\mathrm{C} 3-\mathrm{N} 2-\mathrm{C} 4$ & $118.11(19)$ & $\mathrm{C} 6-\mathrm{C} 7-\mathrm{H} 7$ & 119.6 \\
\hline $\mathrm{C} 1-\mathrm{N} 1-\mathrm{H} 1 \mathrm{~N}$ & $124.6(17)$ & $\mathrm{C} 8-\mathrm{C} 7-\mathrm{H} 7$ & 119.6 \\
\hline $\mathrm{C} 1-\mathrm{N} 1-\mathrm{H} 1 \mathrm{P}$ & $117.5(17)$ & $\mathrm{C} 9-\mathrm{C} 8-\mathrm{C} 7$ & $120.2(2)$ \\
\hline $\mathrm{H} 1 \mathrm{~N}-\mathrm{N} 1-\mathrm{H} 1 \mathrm{P}$ & $118(2)$ & $\mathrm{C} 9-\mathrm{C} 8-\mathrm{H} 8$ & 119.9 \\
\hline $\mathrm{O} 1-\mathrm{C} 1-\mathrm{N} 1$ & $122.2(2)$ & $\mathrm{C} 7-\mathrm{C} 8-\mathrm{H} 8$ & 119.9 \\
\hline $\mathrm{O} 1-\mathrm{C} 1-\mathrm{C} 2$ & $119.3(2)$ & $\mathrm{C} 8-\mathrm{C} 9-\mathrm{C} 10$ & $120.5(2)$ \\
\hline $\mathrm{N} 1-\mathrm{C} 1-\mathrm{C} 2$ & 118.55 (19) & $\mathrm{C} 8-\mathrm{C} 9-\mathrm{H} 9$ & 119.7 \\
\hline $\mathrm{C} 14-\mathrm{C} 2-\mathrm{C} 3$ & $117.0(2)$ & $\mathrm{C} 10-\mathrm{C} 9-\mathrm{H} 9$ & 119.7 \\
\hline $\mathrm{C} 14-\mathrm{C} 2-\mathrm{C} 1$ & $119.62(19)$ & $\mathrm{C} 5-\mathrm{C} 10-\mathrm{C} 9$ & $119.1(2)$ \\
\hline $\mathrm{C} 3-\mathrm{C} 2-\mathrm{C} 1$ & $123.4(2)$ & $\mathrm{C} 5-\mathrm{C} 10-\mathrm{C} 11$ & $119.4(2)$ \\
\hline $\mathrm{N} 2-\mathrm{C} 3-\mathrm{C} 2$ & $125.0(2)$ & $\mathrm{C} 9-\mathrm{C} 10-\mathrm{C} 11$ & $121.5(2)$ \\
\hline $\mathrm{N} 2-\mathrm{C} 3-\mathrm{H} 3$ & 117.5 & $\mathrm{C} 12-\mathrm{C} 11-\mathrm{C} 10$ & $121.5(2)$ \\
\hline $\mathrm{C} 2-\mathrm{C} 3-\mathrm{H} 3$ & 117.5 & $\mathrm{C} 12-\mathrm{C} 11-\mathrm{H} 11$ & 119.3 \\
\hline $\mathrm{N} 2-\mathrm{C} 4-\mathrm{C} 13$ & $121.5(2)$ & $\mathrm{C} 10-\mathrm{C} 11-\mathrm{H} 11$ & 119.3 \\
\hline $\mathrm{N} 2-\mathrm{C} 4-\mathrm{C} 5$ & $118.57(19)$ & $\mathrm{C} 11-\mathrm{C} 12-\mathrm{C} 13$ & $121.0(2)$ \\
\hline $\mathrm{C} 13-\mathrm{C} 4-\mathrm{C} 5$ & $119.92(19)$ & $\mathrm{C} 11-\mathrm{C} 12-\mathrm{H} 12$ & 119.5 \\
\hline
\end{tabular}


supporting information

$\begin{array}{llll}\mathrm{C} 6-\mathrm{C} 5-\mathrm{C} 10 & 119.0(2) & \mathrm{C} 13-\mathrm{C} 12-\mathrm{H} 12 & 119.5 \\ \mathrm{C} 6-\mathrm{C} 5-\mathrm{C} 4 & 122.1(2) & \mathrm{C} 14-\mathrm{C} 13-\mathrm{C} 4 & 118.1(2) \\ \mathrm{C} 10-\mathrm{C} 5-\mathrm{C} 4 & 118.95(19) & \mathrm{C} 14-\mathrm{C} 13-\mathrm{C} 12 & 122.68(19) \\ \mathrm{C} 7-\mathrm{C} 6-\mathrm{C} 5 & 120.3(2) & \mathrm{C} 4-\mathrm{C} 13-\mathrm{C} 12 & 119.3(2) \\ \mathrm{C} 7-\mathrm{C} 6-\mathrm{H} 6 & 119.9 & \mathrm{C} 2-\mathrm{C} 14-\mathrm{C} 13 & 120.37(19) \\ \mathrm{C} 5-\mathrm{C} 6-\mathrm{H} 6 & 119.9 & \mathrm{C} 2-\mathrm{C} 14-\mathrm{H} 14 & 119.8 \\ \mathrm{C} 6-\mathrm{C} 7-\mathrm{C} 8 & 120.9(2) & \mathrm{C} 13-\mathrm{C} 14-\mathrm{H} 14 & 119.8\end{array}$

Hydrogen-bond geometry $\left(A,{ }^{\circ}\right)$

\begin{tabular}{lllll}
\hline$D-\mathrm{H} \cdots A$ & $D-\mathrm{H}$ & $\mathrm{H} \cdots A$ & $D \cdots A$ & $D-\mathrm{H} \cdots A$ \\
\hline $\mathrm{N} 1-\mathrm{H} 1 N^{\cdots} \cdots \mathrm{N} 2^{\mathrm{i}}$ & $0.97(3)$ & $2.17(3)$ & $3.133(3)$ & $173(2)$ \\
$\mathrm{N} 1-\mathrm{H} 1 P \cdots \mathrm{O} 1^{\mathrm{ii}}$ & $0.93(3)$ & $1.96(3)$ & $2.895(3)$ & $175(3)$ \\
$\mathrm{C} 3-\mathrm{H} 3 \cdots \mathrm{N} 2^{\mathrm{i}}$ & 0.95 & 2.41 & $3.361(3)$ & 174 \\
$\mathrm{C} 7-\mathrm{H} 7 \cdots{ }^{\mathrm{iii}}$ & 0.95 & 2.45 & $3.140(3)$ & 129
\end{tabular}

Symmetry codes: (i) $-x+1, y-1 / 2,-z+1 / 2$; (ii) $-x+1,-y-1,-z+1$; (iii) $x,-y+1 / 2, z-1 / 2$. 